\title{
Electromagnetically-induced Deformation of Functionalized Fabric
}

\author{
T.I. Zohdi
}

Received: 24 November 2010 / Published online: 1 February 2011

(C) The Author(s) 2011. This article is published with open access at Springerlink.com

\begin{abstract}
This work investigates the deformation of charged fabric via external electromagnetic fields. A reduced-order model is constructed by combining a yarn-segment network representation with dynamic discrete/lumped charged-masses. The deformation of the fabric is dictated by solving a coupled system of differential equations for the motion of the lumped charged-masses, which are coupled through the yarn-segments. Initially, the effects of the electric and magnetic fields are analytically studied for the components (yarnsegment network and charged-masses) that comprise the model. Quantitative numerical simulations are then provided for the entire, assembled, larger-scale, coupled system, based on a computationally-efficient time-stepping algorithm. The model is relatively easy to implement and provides analysts with a straightforward tool to study electromagnetic fabric systems.
\end{abstract}

Keywords Fabric networks · Electromagnetics

Mathematics Subject Classification (2000) 74K05 - 74K35 · 74F15

\section{Reduced Order Fabric Network Model}

This work studies the deformation of an electromagnetically-sensitive fabric (textile) structure, induced by external electric and magnetic fields. There are many applications for such materials, for example electromagnetic actuators, micro-electromechanical systems (MEMS), etc. We assume that the fabric can carry a charge. One way of achieving this is by adding charged fine-scale particles to a polymer matrix, for example using ion-implantation

Dedicated to Prof. Don Carlson, who inspired a generation of young mechanicians with his quest for scientific truth and rigor.

Submitted to a special issue in honor Prof. Donald Carlson.

T.I. Zohdi $(\bowtie)$

Department of Mechanical Engineering, University of California, 6117 Etcheverry Hall, Berkeley, CA 94720-1740, USA

e-mail: zohdi@me.berkeley.edu 

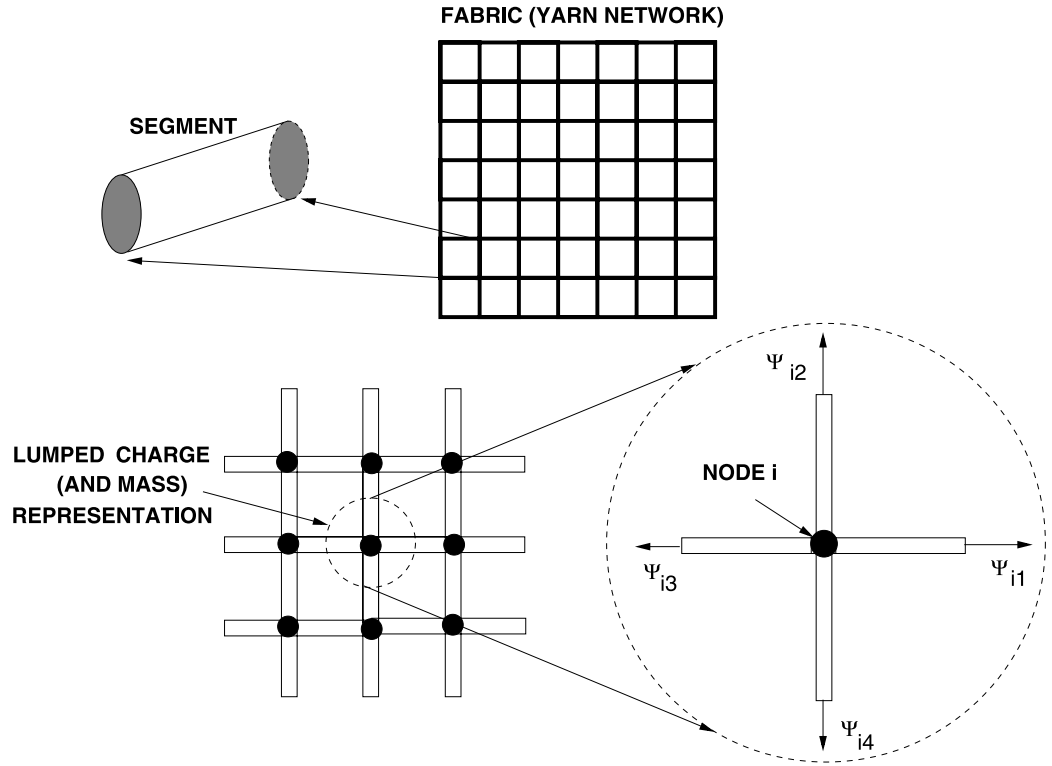

Fig. 1 A network representation of woven-fabric by coupled yarn-segments. The yarn-segments are joined together by pin-joints to form a network, and the mass and electrical charge of the material are lumped at the connection points

processing techniques, from which the (nominal) yarn (Fig. 1) is fabricated. The present study investigates the connection between the electromagnetic loading and fabric actuation, building on the recent analysis of Zohdi [46], which focused on a specific application involving electromagnetic ballistic fabric shielding, whereby the Lorentz force was harnessed to enhance material resistance capabilities beyond purely mechanical effects, in a projectile contact/impact loading regime. Here, we focus more deeply on what effects an external electromagnetic field has on such fabrics. We model the entire structure (Fig. 1) by combining a fabric network model with discrete/lumped charged-masses. The charges are assumed to be fixed (implanted) in the fabric. Dynamically running current through the fabric is not considered in this work, however, some comments on such an approach are given at the end of this work. As a model problem, we consider an undeformed (initially planar) yarn-segment network representation of a woven fabric (Fig. 1), and make two primary simplifying assumptions: (1) the yarn-segments are pin-joined at the nodes, producing no moments, to form a network and (2) the electrically-charged state of the fabric is represented by discrete lumped "charged-masses" whose locations coincide with those of the pin-joints. The deformation of the fabric is dictated by solving a ("yarn-coupled") system of differential equations for the motion of the interconnected lumped charged-masses.

Remark The "functionalization" or "tailoring" of materials by the addition of fine-scale particles ("particulates") is a process that has a long history in engineering. The usual approach is to add particulates that possess a desired property to enhance a base (binder) material. The development of mathematical (homogenization) models to determine the resulting effective properties of such materials dates back to the 1800s, for example Maxwell [23, 24] and Lord Rayleigh [30], to name a few. There are many homogenization techniques available in the literature, which seek to estimate the aggregate responses microheterogeneous 
materials. For a thorough analysis of many of such methods, see Torquato [34], Jikov et al. [19], Hashin [17] and Nemat-Nasser and Hori [26] for solid-mechanics oriented treatments and Zohdi and Wriggers [48] for computational aspects. For a series of works on continuum modeling and finite-element simulation of the deformation of magnetoelastic functionalized membranes and films (for example mixtures of iron powder and polydimethlsiloxane), we refer the reader to recent studies by Barham et al. [4-7]. Applications for such materials are driven by the extensive sensor, actuator and MEMS industries. See, for example, Rebeiz et al. [32], Quandt and Ludwig [31], Grimes et al. [13] and Kouzoudis and Grimes [21, 22], Azevedo et al. [3], Jones et al. [20] and Myers et al. [25] for specific applications.

\section{Yarn-segment Network Representation of Fabric}

We assume that the compressive response of the fabric (yarn-segment) is insignificant for the applications under consideration, and employ a so-called relaxed model, whereby a zero stress state is enforced for a compressive state. For details on a wide variety of relaxed models, we refer the reader to works dating back to Pipkin [28], Buchholdt et al. [8], Pangiotopoulos [27], Bufler and Nguyen-Tuong [9] and Cannarozzi [10, 11], Steigmann [33], Haseganu and Steigmann [14-16] and Atai and Steigmann [1,2]. Relaxed formulations have served as a foundation for computational models describing rupture of ballistic fabric shielding in Zohdi [36, 43, 46], Zohdi and Steigmann [37], Zohdi and Powell [41] and Powell and Zohdi [29] and are the basis for the present approach.

In addition to the previously mentioned assumptions, we further assume that: (1) the yarn-segments are quite thin, experiencing a uniaxial-stress condition, whereby the forces only act along the length of the yarn-segments, (2) the yarn-segments remain straight, undergoing a homogeneous (axial) stress state and (3) yarn-segment buckling phenomena is insignificant. We write one-dimensional constitutive laws in terms of the Piola-Kirchhoff stresses (mimicing 3-D approaches), defined by

$$
P=\frac{\text { force on referential area }}{\text { referential area }},
$$

and then transform the result to the second Piola-Kirchhoff stress via $P=U S$, where $U=$ $\frac{L}{L_{o}}$ is the stretch ratio, $L$ is the deformed length of the yarn-segment, $L_{o}$ is its original length and where we note that for a relaxed model, when $U \leq 1$ (compression), we enforce $P=0$. A standard constitutive relation $S=\mathcal{F}(U)$ is then employed, with the primary objective being to extract the force carried in the yarn-segment $\left(\boldsymbol{\psi}^{\text {yarn }}\right)$, which is needed later for the dynamics of the lumped charged-masses. Specifically,

$$
P=\frac{\psi^{\text {yarn }}}{A_{o}} \Rightarrow \psi^{\text {yarn }}=U S A_{o}=\frac{L}{L_{o}} S A_{o} .
$$

We shall adopt a simple one-dimensional model for the stored energy, $W=\frac{1}{2} \mathbb{E} \mathcal{E}^{2}$, where $\mathbb{E}$ is Young's modulus and $\mathcal{E} \stackrel{\text { def }}{=} \frac{1}{2}\left(U^{2}-1\right)$ is the Green-Lagrange strain, with the second Piola-Kirchhoff stress given by $\frac{\partial W}{\partial \mathcal{E}}=S=\mathbb{E} \mathcal{E}$. Thus, for the yarn-segment,

$$
P=\frac{\psi^{\text {yarn }}}{A_{o}} \Rightarrow \psi^{\text {yarn }}=U S A_{o}=\frac{L}{L_{o}} S A_{o}=\frac{L}{2 L_{o}} \mathbb{E}\left(\left(\frac{L}{L_{o}}\right)^{2}-1\right) A_{o} .
$$




\section{Charged-mass Motion}

The dynamics of the lumped charged-masses are given by

$$
m_{i} \ddot{\boldsymbol{r}}_{i}=\underbrace{\boldsymbol{\psi}_{i}^{\text {tot }}}_{\text {total }}=\underbrace{\boldsymbol{\psi}_{i}^{\text {em }}}_{\text {electromagnetic forces }}+\underbrace{\sum_{I=1}^{4} \boldsymbol{\psi}_{i I}^{\text {yarn }}}_{\text {surrounding yarn }},
$$

where $i=1,2, \ldots, N$, where $N$ is the number of lumped charged-masses, $\boldsymbol{\psi}_{i}^{e m}$ represents the electromagnetic contribution, $\boldsymbol{\psi}_{i I}^{\text {yarn }}$ represents the contributions of the four yarn intersecting at charged-mass $i$ (Fig. 1) and $m_{i}$ is the mass of a single lumped charged-mass (the total fabric mass divided by the total number of charged-masses). The forces from the $I$ th surrounding yarn-segment (there are four of them for the type of rectangular weaving pattern considered) acting on the $i$ th lumped charged-mass is $\boldsymbol{\psi}_{i I}^{y \text { arn }}=U_{I} S_{I} A_{o} \boldsymbol{a}_{i I}$ ( $A_{o}$ is the undeformed cross-sectional area of the yarn), where the unit axial yarn direction is given by $\boldsymbol{a}_{i I}=\frac{\boldsymbol{r}_{I}^{+}-\boldsymbol{r}_{I}^{-}}{\left\|\boldsymbol{r}_{I}^{+}-\boldsymbol{r}_{I}^{-}\right\|}$, where $\boldsymbol{r}_{I}^{+}$denotes the position vector of the endpoint connected to the lumped charged-mass and $\boldsymbol{r}_{I}^{-}$denotes the endpoint that is connected to it neighboring charged-mass (Fig. 1). ${ }^{1}$ Clearly, $\boldsymbol{\psi}_{i I}^{\text {yarn }}$ is a function of the charged-mass positions $\left(\boldsymbol{r}_{i}\right)$, which are all coupled together, leading to a system of equations. In order to solve the resulting coupled system, we develop an iterative solution scheme later in the presentation.

To describe the electromagnetic behavior of the lumped charges $\left(q_{i}\right) /$ masses $\left(m_{i}\right)$ in the model, recall the main observations in conjunction with electromagnetic phenomena (Jackson [18]), namely (a) in the presence of an external electric field, $\boldsymbol{E}^{\text {ext }}$, a point charge $q$ experiences a force $\boldsymbol{\psi}^{e}=q \boldsymbol{E}^{e x t}$ and (b) in the presence of an external magnetic field, $\boldsymbol{B}^{\text {ext }}$, if a point charge is moving with velocity $\boldsymbol{v}$, then the point charge experiences a force $\boldsymbol{\psi}^{m}=q \boldsymbol{v} \times \boldsymbol{B}$. Thus, if a (point) charged-mass is moving through the region possessing both electric and magnetic fields, then

$$
\boldsymbol{\psi}_{i}^{e m}=\boldsymbol{\psi}_{i}^{e}+\boldsymbol{\psi}_{i}^{m} \stackrel{\text { def }}{=} q_{i}\left(\boldsymbol{E}^{e x t}+\boldsymbol{v}_{i} \times \boldsymbol{B}^{e x t}\right) .
$$

Here, we assume that $\boldsymbol{E}^{\text {ext }}$ and $\boldsymbol{B}^{\text {ext }}$ are static, uncoupled and independently controllable. In other words, the external fields are assumed to be independent of the response of the system (dead loading). Furthermore, for the problems of interest here, we assume that the electromagnetic interaction between charged-masses is negligible, relative to the externallyapplied field. For detailed charged-mass interaction formulations and simulation techniques, we refer the reader to Zohdi [38-45].

\section{Qualitative Dependency of Network Deformation on Electromagnetics}

Consider purely electrical loading (no magnetic field), and a single charged-mass joined to four pieces of yarn-segments, all of which are secured to a rigid foundation (Fig. 2).

\footnotetext{
${ }^{1}\|\cdot\|$ indicates the Euclidean norm in $R^{3}$.
} 
Fig. 2 A single yarn-unit. LEFT: top view and RIGHT: side view

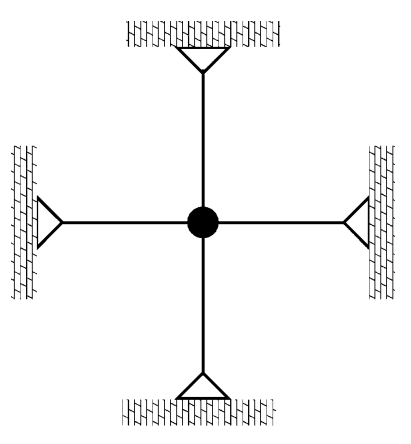

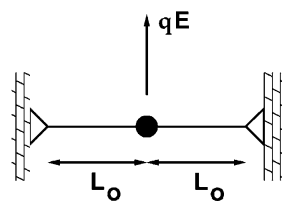

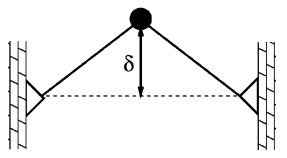

Performing a static force balance yields

$$
\begin{aligned}
& q E^{e x t}=2 P A_{o} \frac{\delta}{L}=2 U S A_{o} \frac{\delta}{L}=\frac{L}{L_{o}} \mathbb{E}\left(\frac{L^{2}}{L_{o}^{2}}-1\right) A_{o} \delta=\mathbb{E} A_{o} \frac{\delta^{3}}{L_{o}^{3}} \\
& \Rightarrow \delta=L_{o}\left(\frac{q E^{e x t}}{A_{o} \mathbb{E}}\right)^{1 / 3} .
\end{aligned}
$$

The key observation is that the deformation $(\delta)$ depends linearly on the original length, $L_{o}$, has an cube-root (sublinear) dependency on the product of the charge and electric field, and has an inverse-cubic dependency on the product of the original cross-sectional area and Young's modulus.

Remark For the magnetic fields to induce a comparable deformation, one must have

$$
\left\|\boldsymbol{E}^{e x t}\right\| \approx\left\|\boldsymbol{v} \times \boldsymbol{B}^{e x t}\right\| \leq\|\boldsymbol{v}\|\left\|\boldsymbol{B}^{e x t}\right\|,
$$

thus $\left\|\boldsymbol{B}^{\text {ext }}\right\| \geq \frac{\left\|\boldsymbol{E}^{e x t}\right\|}{\|\boldsymbol{v}\|}$. In order to understand the electrically- and magnetically-induced tendencies on the system, we first study an isolated charged-mass under the influence of an electromagnetic field, next.

\section{Induced Isolated (Unconstrained) Charged-mass Motion}

We consider an isolated lumped charged mass (Fig. 3) with position vector denoted by $\boldsymbol{r}$ $(\dot{\boldsymbol{r}}=\boldsymbol{v}, \ddot{\boldsymbol{r}}=\dot{\boldsymbol{v}})$, whose unconstrained motion is governed by

$$
m \dot{\boldsymbol{v}}=q\left(\boldsymbol{E}^{e x t}+\boldsymbol{v} \times \boldsymbol{B}^{e x t}\right) .
$$

The governing (8), written in component form is, for the $x_{1}$-component

$$
\dot{v}_{1}=\frac{q}{m}\left(E_{1}^{e x t}+\left(v_{2} B_{3}^{e x t}-v_{3} B_{2}^{e x t}\right)\right),
$$

for the $x_{2}$-component

$$
\dot{v}_{2}=\frac{q}{m}\left(E_{2}^{e x t}-\left(v_{1} B_{3}^{\text {ext }}-v_{3} B_{1}^{\text {ext }}\right)\right),
$$


Fig. 3 An isolated charged-mass with an applied "dead" electromagnetic field for special cases \# 1 and \# 2

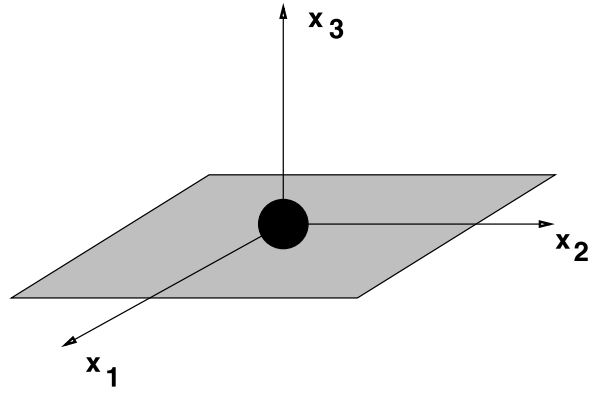

and for the $x_{3}$-component

$$
\dot{v}_{3}=\frac{q}{m}\left(E_{3}^{e x t}+\left(v_{1} B_{2}^{e x t}-v_{2} B_{1}^{e x t}\right)\right) \text {, }
$$

where $\boldsymbol{E}^{\text {ext }}=\left(E_{1}^{\text {ext }}, E_{2}^{\text {ext }}, E_{3}^{\text {ext }}\right)$ is an independent external electric field (not dependent on the charged-masses ("dead")) and $\boldsymbol{B}^{\text {ext }}=\left(B_{1}^{\text {ext }}, B_{2}^{\text {ext }}, B_{3}^{\text {ext }}\right)$ is an independent external magnetic field. These coupled equations can be solved analytically for an isolated charged-mass. We consider two relevant cases.

\subsection{Special Case \# 1: No Magnetic Field $\left(\boldsymbol{E}^{e x t}=E_{3}^{e x t} \boldsymbol{e}_{3}\right.$ and $\left.\boldsymbol{B}^{e x t}=\boldsymbol{0}\right)$}

In the special case when there is no magnetic field, if $\boldsymbol{r}(t=0)=\mathbf{0}, \boldsymbol{v}(t=0)=\mathbf{0}, \boldsymbol{B}^{\text {ext }}=\mathbf{0}$ and $\boldsymbol{E}^{\text {ext }}=E_{3}^{\text {ext }} \boldsymbol{e}_{3}$, the solution for the dynamics of an isolated charged-mass is

$$
\left[\begin{array}{l}
v_{1}(t) \\
v_{2}(t) \\
v_{3}(t)
\end{array}\right]=\left[\begin{array}{c}
0 \\
0 \\
\frac{q}{m} E_{3}^{e x t} t
\end{array}\right] \Rightarrow\left[\begin{array}{c}
r_{1}(t) \\
r_{2}(t) \\
r_{3}(t)
\end{array}\right]=\left[\begin{array}{c}
0 \\
0 \\
\frac{q}{2 m} E_{3}^{e x t} t^{2}
\end{array}\right] .
$$

5.2 Special Case \# 2: Combined Electric and Magnetic Fields $\left(\boldsymbol{E}^{e x t}=E_{3}^{e x t} \boldsymbol{e}_{3}\right.$ and $\left.\boldsymbol{B}^{\text {ext }}=B_{1}^{\text {ext }} \boldsymbol{e}_{1}\right)$

Now consider both the electric and magnetic fields to be present, $\boldsymbol{r}(t=0)=\mathbf{0}, \boldsymbol{v}(t=0)=\mathbf{0}$, $\boldsymbol{B}^{\text {ext }}=B_{1}^{\text {ext }} \boldsymbol{e}_{1}$ and $\boldsymbol{E}^{\text {ext }}=E_{3}^{\text {ext }} \boldsymbol{e}_{3}$, consequently, for an isolated charged-mass

$$
\left[\begin{array}{l}
v_{1}(t) \\
v_{2}(t) \\
v_{3}(t)
\end{array}\right]=\left[\begin{array}{c}
0 \\
\left(\frac{E_{3}^{\text {ext }}}{B_{1}^{\text {ext }}}\right)(1-\cos \omega t) \\
\left(\frac{E_{3}^{\text {ext }}}{B_{1}^{\text {ext }}}\right) \sin \omega t
\end{array}\right] \Rightarrow\left[\begin{array}{c}
r_{1}(t) \\
r_{2}(t) \\
r_{3}(t)
\end{array}\right]=\left[\begin{array}{c}
0 \\
\left(\frac{E_{3}^{\text {ext }}}{B_{1}^{\text {ext }}}\right)\left(t-\frac{\sin \omega t}{\omega}\right) \\
\left(\frac{E_{3}^{\text {xxt }}}{B_{1}^{\text {ext }} \omega}\right)(1-\cos \omega t)
\end{array}\right],
$$

where $\omega=\frac{q B_{1}^{\text {ext }}}{m}$ is known as the cyclotron frequency. The cyclotron frequency (gyrofrequency) is the angular frequency at which a charged-mass makes circular orbits in a plane perpendicular to the static magnetic field (see Jackson [18] for general comments and Zohdi [45] for specific applications to particulate flows). The $x_{1}$-magnetic field causes a "pull" in the $x_{2}$-direction, provided that there is an $x_{3}$-component of the electric field. We remark that as $B_{1}^{\text {ext }} \rightarrow 0$ then the solutions in (13) converge to (12), which can be proven by repeated us of L'Hospital's rule. General solutions are considered next. 


\subsection{General Solutions: Magnetic Rotation Axes}

The coupling term $\boldsymbol{v} \times \boldsymbol{B}^{\text {ext }}$, which can explicitly be written in the following matrix form:

$$
\boldsymbol{v} \times \boldsymbol{B}^{\text {ext }}=\left[\begin{array}{ccc}
0 & B_{3}^{\text {ext }} & -B_{2}^{\text {ext }} \\
-B_{3}^{\text {ext }} & 0 & B_{1}^{\text {ext }} \\
B_{2}^{\text {ext }} & -B_{1}^{\text {ext }} & 0
\end{array}\right]\left[\begin{array}{l}
v_{1} \\
v_{2} \\
v_{3}
\end{array}\right] \stackrel{\text { def }}{=} \boldsymbol{A} \cdot \boldsymbol{v} .
$$

The system of equations can be written as

$$
\dot{\boldsymbol{v}}-\frac{q}{m} \boldsymbol{A} \cdot \boldsymbol{v}=\frac{q}{m} \boldsymbol{E}
$$

We define an "eigensystem" via

$$
\boldsymbol{v}=\boldsymbol{T} \cdot \hat{\boldsymbol{v}}
$$

and insert into the governing equations to yield

$$
\dot{\hat{\boldsymbol{v}}}-\frac{q}{m} \underbrace{\left(\boldsymbol{T}^{-1} \cdot \boldsymbol{A} \cdot \boldsymbol{T}\right)}_{\boldsymbol{K}} \hat{\boldsymbol{v}}=\frac{q}{m} \boldsymbol{T}^{-1} \cdot \boldsymbol{E} .
$$

The proper choice of $\boldsymbol{T}$ to decouple the system is to form $\boldsymbol{T}$ from the eigenvectors of $\boldsymbol{A}$. The eigenvalues are computed from

$$
\begin{aligned}
\boldsymbol{v} & \times \boldsymbol{B}^{\text {ext }}=\left|\begin{array}{ccc}
-\lambda & B_{3}^{\text {ext }} & -B_{2}^{\text {ext }} \\
-B_{3}^{\text {ext }} & -\lambda & B_{1}^{\text {ext }} \\
B_{2}^{\text {ext }} & -B_{1}^{\text {ext }} & -\lambda
\end{array}\right|=0 \\
\Rightarrow \lambda_{1} & =0, \lambda_{2}=-i\left\|\boldsymbol{B}^{\text {ext }}\right\|, \lambda_{3}=+i\left\|\boldsymbol{B}^{\text {ext }}\right\| .
\end{aligned}
$$

For the first eigenvector $\left(\lambda_{1}=0\right)$

$$
\boldsymbol{\Lambda}^{(1)}=\frac{1}{\left\|\boldsymbol{B}^{\text {ext }}\right\|}\left[\begin{array}{c}
B_{1}^{\text {ext }} \\
B_{2}^{\text {ext }} \\
B_{3}^{\text {ext }}
\end{array}\right]
$$

For the second eigenvector $\left(\lambda_{2}=-i\left\|\boldsymbol{B}^{\text {ext }}\right\|\right)$

$$
\boldsymbol{\Lambda}^{(2)}=\frac{1}{\gamma}\left[\begin{array}{c}
B_{1}^{\text {ext }} B_{2}^{\text {ext }}-i B_{3}^{\text {ext }}\left\|\boldsymbol{B}^{\text {ext }}\right\| \\
-\left(B_{1}^{\text {ext }}\right)^{2}-\left(B_{3}^{\text {ext }}\right)^{2} \\
B_{2}^{\text {ext }} B_{3}^{\text {ext }}+i B_{1}^{\text {ext }}\left\|\boldsymbol{B}^{\text {ext }}\right\|
\end{array}\right],
$$

where

$$
\gamma=\sqrt{\left|\left(B_{1}^{\text {ext }}\right)^{2}+\left(B_{3}^{\text {ext }}\right)^{2}\right|^{2}+\left|i B_{1}^{\text {ext }}\left\|\boldsymbol{B}^{\text {ext }}\right\|+B_{2}^{\text {ext }} B_{3}^{\text {ext }}\right|^{2}+\left|B_{1}^{\text {ext }} B_{2}^{\text {ext }}-i B_{3}^{\text {ext }}\left\|\boldsymbol{B}^{\text {ext }}\right\|\right|^{2}} .
$$

For the third eigenvector $\left(\lambda_{2}=i\left\|\boldsymbol{B}^{e x t}\right\|\right)$

$$
\boldsymbol{\Lambda}^{(3)}=\frac{1}{\hat{\gamma}}\left[\begin{array}{c}
B_{1}^{\text {ext }} B_{2}^{\text {ext }}+i B_{3}^{\text {ext }}\left\|\boldsymbol{B}^{\text {ext }}\right\| \\
-\left(B_{1}^{\text {ext }}\right)^{2}-\left(B_{3}^{\text {ext }}\right)^{2} \\
B_{2}^{\text {ext }} B_{3}^{\text {ext }}-i B_{1}^{\text {ext }}\left\|\boldsymbol{B}^{\text {ext }}\right\|
\end{array}\right]
$$


where

$$
\hat{\gamma}=\sqrt{\left|-\left(B_{1}^{\text {ext }}\right)^{2}-\left(B_{3}^{\text {ext }}\right)^{2}\right|^{2}+\left|B_{1}^{\text {ext }} B_{2}^{\text {ext }}+i B_{3}^{\text {ext }}\left\|\boldsymbol{B}^{\text {ext }}\right\|\right|^{2}+\left|B_{2}^{\text {ext }} B_{3}^{\text {ext }}-i B_{1}^{\text {ext }}\left\|\boldsymbol{B}^{\text {ext }}\right\|\right|^{2} .}
$$

The first eigenvector indicates the direction of the axis of rotation, while the second and third (complex-conjugate) eigenvalues dictate a (cyclotron) frequency and a radius of a helical circle. See Zohdi [45] for an analysis of trajectories and Jackson [18] for more general treatments.

One can decouple the coupled (vector component) system by forming a matrix from the following set of eigenvectors:

$$
\left[\begin{array}{c}
\Lambda_{1}^{(1)} \\
\Lambda_{2}^{(1)} \\
\Lambda_{3}^{(1)}
\end{array}\right]_{\lambda=\lambda_{1}},\left[\begin{array}{c}
\Lambda_{1}^{(2)} \\
\Lambda_{2}^{(2)} \\
\Lambda_{3}^{(2)}
\end{array}\right]_{\lambda=\lambda_{2}},\left[\begin{array}{c}
\Lambda_{1}^{(3)} \\
\Lambda_{2}^{(3)} \\
\Lambda_{3}^{(3)}
\end{array}\right]_{\lambda=\lambda_{3}} .
$$

Performing a similarity transform to decouple the system, we obtain

$$
\begin{gathered}
{\left[\begin{array}{lll}
1 & 0 & 0 \\
0 & 1 & 0 \\
0 & 0 & 1
\end{array}\right]\left[\begin{array}{c}
\dot{\hat{v}}_{1} \\
\dot{\hat{v}}_{2} \\
\dot{\hat{v}}_{3}
\end{array}\right]-\frac{q}{m}\left[\begin{array}{ccc}
0 & 0 & 0 \\
0 & i\left\|\boldsymbol{B}^{\text {ext }}\right\| & 0 \\
0 & 0 & -i\left\|\boldsymbol{B}^{\text {ext }}\right\|
\end{array}\right]\left\{\begin{array}{l}
\hat{v}_{1} \\
\hat{v}_{2} \\
\hat{v}_{3}
\end{array}\right\}} \\
=\frac{q}{m}\left[\begin{array}{ccc}
\Lambda_{1}^{(1)} & \Lambda_{1}^{(2)} & \Lambda_{1}^{(3)} \\
\Lambda_{2}^{(1)} & \Lambda_{2}^{(2)} & \Lambda_{2}^{(3)} \\
\Lambda_{3}^{(1)} & \Lambda_{3}^{(2)} & \Lambda_{3}^{(3)}
\end{array}\right]^{-1}\left\{\begin{array}{l}
E_{1}^{\text {ext }} \\
E_{2}^{\text {ext }} \\
E_{3}^{\text {ext }}
\end{array}\right\} \stackrel{\text { def }}{=}\left\{\begin{array}{l}
\hat{f}_{1} \\
\hat{f}_{2} \\
\hat{f}_{3}
\end{array}\right\} .
\end{gathered}
$$

The decoupled problems can be written as

$$
\begin{aligned}
\dot{\hat{v}}_{1} & =\hat{f}_{1}, \\
\dot{\hat{v}}_{2}-i\left\|\boldsymbol{B}^{e x t}\right\| \hat{v}_{2} & =\hat{f}_{2}, \\
\dot{\hat{v}}_{3}+i\left\|\boldsymbol{B}^{\text {ext }}\right\| \hat{v}_{3} & =\hat{f}_{3},
\end{aligned}
$$

and can be solved individually (decoupled). Afterward, the solution in the transformed space are transformed back to yield:

$$
\left[\begin{array}{ccc}
\Lambda_{1}^{(1)} & \Lambda_{1}^{(2)} & \Lambda_{1}^{(3)} \\
\Lambda_{2}^{(1)} & \Lambda_{2}^{(2)} & \Lambda_{2}^{(3)} \\
\Lambda_{3}^{(1)} & \Lambda_{3}^{(2)} & \Lambda_{3}^{(3)}
\end{array}\right]\left\{\begin{array}{c}
\hat{v}_{1} \\
\hat{v}_{2} \\
\hat{v}_{3}
\end{array}\right\}=\left\{\begin{array}{l}
v_{1} \\
v_{2} \\
v_{3}
\end{array}\right\}
$$

Remark On can interpret the preceding analysis as a study of one part of the total influence on the motion of the lumped charged-masses by considering a decomposition of the forces governing the dynamics of the lumped-charged-masses into an independent "external" part and a charged-mass-to-charged-mass "internal" (fabric-interaction) part

$$
\underbrace{q_{i}\left(\boldsymbol{E}^{e x t}+\boldsymbol{v}_{i} \times \boldsymbol{B}^{e x t}\right)+\sum_{j \neq i}^{N} \boldsymbol{\Psi}_{i j}}_{\text {total }}=\underbrace{q_{i}\left(\boldsymbol{E}^{e x t}+\boldsymbol{v}_{i} \times \boldsymbol{B}^{e x t}\right)}_{\text {external }}+\underbrace{\sum_{j \neq i}^{N} \boldsymbol{\Psi}_{i j}}_{\text {internal-fabric-coupling }}
$$


For fabric-coupled multiple masses undergoing dynamic motion due to combined electromagnetic fields, we must employ numerical methods, which we discuss next.

\section{Numerical Solution Scheme for Fabric-interacting Lumped Charged-masses}

Following Zohdi [46], in order describe the overall time-stepping scheme, we first start with the dynamics of a single ( $i$ th) lumped charged-mass. The equation of motion is given by

$$
m_{i} \dot{\boldsymbol{v}}_{i}=\boldsymbol{\psi}_{i}^{\text {tot }}
$$

where $\boldsymbol{\psi}_{i}^{\text {tot }}$ is the total force provided from interactions with the external environment (yarn, electromagnetics, etc.). Employing the trapezoidal-like rule $(0 \leq \phi \leq 1)$

$\boldsymbol{r}_{i}(t+\Delta t)=\boldsymbol{r}_{i}(t)+\boldsymbol{v}_{i}(t) \Delta t+\frac{\phi(\Delta t)^{2}}{m_{i}}\left(\phi \boldsymbol{\psi}_{i}^{t o t}\left(\boldsymbol{r}_{i}(t+\Delta t)\right)+(1-\phi) \boldsymbol{\psi}_{i}^{t o t}\left(\boldsymbol{r}_{i}(t)\right)\right)+\hat{\mathcal{O}}(\Delta t)^{2}$,

where if $\phi=1$, then (32) becomes the (implicit) Backward Euler scheme, which is very stable, dissipative and $\hat{\mathcal{O}}(\Delta t)^{2}=\mathcal{O}(\Delta t)^{2}$ locally in time, if $\phi=0$, then (32) becomes the (explicit) Forward Euler scheme, which is conditionally stable and $\hat{\mathcal{O}}(\Delta t)^{2}=\mathcal{O}(\Delta t)^{2}$ locally in time and if $\phi=0.5$, then (32) becomes the (implicit) Midpoint scheme, which is stable and $\hat{\mathcal{O}}(\Delta t)^{2}=\mathcal{O}(\Delta t)^{3}$ locally in time. ${ }^{2}$ Equation (32) can be solved recursively by recasting the relation as

$$
\boldsymbol{r}_{i}^{L+1, K}=\mathcal{G}\left(\boldsymbol{r}_{i}^{L+1, K-1}\right)+\mathcal{R}_{i},
$$

where $K=1,2,3, \ldots$, is the index of iteration within time step $L+1$ and $\mathcal{R}_{i}$ is a remainder term that does not depend on the solution, i.e. $\mathcal{R}_{i} \neq \mathcal{R}_{i}\left(\boldsymbol{r}_{1}^{L+1}, \boldsymbol{r}_{2}^{L+1}, \ldots, \boldsymbol{r}_{N}^{L+1}\right)$. The convergence of such a scheme is dependent on the behavior of $\mathcal{G}$. Namely, a sufficient condition for convergence is that $\mathcal{G}$ is a contraction mapping for all $\boldsymbol{r}_{i}^{L+1, K}, K=1,2,3, \ldots$, In order to investigate this further, we define the iteration error as

$$
\varpi_{i}^{L+1, K} \stackrel{\text { def }}{=} \boldsymbol{r}_{i}^{L+1, K}-\boldsymbol{r}_{i}^{L+1}
$$

A necessary restriction for convergence is iterative self consistency, i.e. the "exact" (discretized) solution must be represented by the scheme

$$
\mathcal{G}\left(\boldsymbol{r}_{i}^{L+1}\right)+\mathcal{R}_{i}=\boldsymbol{r}_{i}^{L+1}
$$

Enforcing this restriction, a sufficient condition for convergence is the existence of a contraction mapping

$$
\|\underbrace{\boldsymbol{r}_{i}^{L+1, K}-\boldsymbol{r}_{i}^{L+1}}_{\varpi_{i}^{L+1, K}}\|=\left\|\mathcal{G}\left(\boldsymbol{r}_{i}^{L+1, K-1}\right)-\mathcal{G}\left(\boldsymbol{r}_{i}^{L+1}\right)\right\| \leq \eta^{L+1, K}\left\|\boldsymbol{r}_{i}^{L+1, K-1}-\boldsymbol{r}_{i}^{L+1}\right\|,
$$

\footnotetext{
${ }^{2}$ In order to streamline the notation, we drop the cumbersome $\mathcal{O}(\Delta t)$-type terms.
} 
where, if $0 \leq \eta^{L+1, K}<1$ for each iteration $K$, then $\varpi_{i}^{L+1, K} \rightarrow \mathbf{0}$ for any arbitrary starting value $\boldsymbol{r}_{i}^{L+1, K=0}$, as $K \rightarrow \infty$. This type of contraction condition is sufficient, but not necessary, for convergence. Explicitly, the recursion is

$$
\begin{aligned}
\boldsymbol{r}_{i}^{L+1, K}= & \underbrace{\boldsymbol{r}_{i}^{L}+\boldsymbol{v}_{i}^{L} \Delta t+\frac{\phi(\Delta t)^{2}}{m_{i}}\left((1-\phi) \boldsymbol{\psi}_{i}^{t o t, L}\right)}_{\mathcal{R}_{i}} \\
& +\underbrace{\frac{\phi(\Delta t)^{2}}{m_{i}}\left(\phi \boldsymbol{\psi}_{i}^{t o t, L+1, K-1}\right)}_{\mathcal{G}\left(\boldsymbol{r}_{i}^{L+1, K-1}\right)},
\end{aligned}
$$

where

$$
\boldsymbol{\psi}_{i}^{t o t, L}=\boldsymbol{\psi}_{i}^{t o t, L}\left(\boldsymbol{r}_{1}^{L}, \boldsymbol{r}_{2}^{L}, \ldots, \boldsymbol{r}_{N}^{L}\right)
$$

and

$$
\boldsymbol{\psi}_{i}^{t o t, L+1, K-1}=\boldsymbol{\psi}_{i}^{t o t, L+1, K-1}\left(\boldsymbol{r}_{1}^{L+1, K-1}, \boldsymbol{r}_{2}^{L+1, K-1}, \ldots, \boldsymbol{r}_{N}^{L+1, K-1}\right) .
$$

According to (36), convergence is scaled by $\eta \propto \frac{(\Delta t)^{2}}{m_{i}}$, and that the contraction constant of $\mathcal{G}$ is (1) directly dependent on the magnitude of the interaction forces, (2) inversely proportional to the lumped masses $m_{i}$ and (3) directly proportional to $\Delta t$. Thus, if convergence is slow within a time step, the time step size, which is adjustable, can be reduced by an appropriate amount to increase the rate of convergence. It is also desirable to simultaneously maximize the time-step sizes to decrease overall computing time, while obeying an error tolerance on the numerical solution's accuracy. In order to achieve this goal, we follow an approach found in Zohdi [38-46], originally developed for continuum thermo-chemical multifield problems where (1) one approximates $\eta^{L+1, K} \approx S(\Delta t)^{p}$ (S is a constant) and (2) one assumes that the error within an iteration to behave according to $\left(S(\Delta t)^{p}\right)^{K} \varpi^{L+1,0}=\varpi^{L+1, K}$, $K=1,2, \ldots$, where $\varpi^{L+1,0}$ is the initial norm of the iterative error and $S$ is intrinsic to the system. ${ }^{3}$ The objective is to meet an error tolerance in exactly a preset number of iterations. To this end, one writes $\left(S\left(\Delta t_{\mathrm{tol}}\right)^{p}\right)^{K_{d}} \varpi^{L+1,0}=T O L$, where $T O L$ is a tolerance and where $K_{d}$ is the number of desired iterations. ${ }^{4}$ If the error tolerance is not met in the desired number of iterations, the contraction constant $\eta^{L+1, K}$ is too large. Accordingly, one can solve for a new smaller step size, under the assumption that $S$ is constant,

$$
\Delta t_{\mathrm{tol}}=\Delta t\left(\frac{\left(\frac{T O L}{\varpi^{L+1,0}}\right)^{\frac{1}{p K}}}{\left(\frac{\varpi^{L+1, K}}{\varpi^{L+1,0}}\right)^{\frac{1}{p K}}}\right) .
$$

The assumption that $S$ is constant is not crucial, since the time steps are to be recursively refined and unrefined throughout the simulation. The expression in (39) can also be used for time step enlargement, to reduce computational effort, if convergence is met in less than $K_{d}$ iterations. Numerous parameter studies of this algorithm can be found in Zohdi [38-46].

\footnotetext{
${ }^{3}$ For the class of problems under consideration, due to the quadratic dependency on $\Delta t, p \approx 2$.

${ }^{4}$ Typically, $K_{d}$ is chosen to be between five to ten iterations.
} 
An implementation of the procedure is as follows:

(1) GLOBAL FIXED-POINT ITERATION: (SET $i=1$ AND $K=0$ ):

(2) IF $i>N$ THEN GO TO (4) $\quad(N=$ \# OF CHARGED-MASSES $)$

(3) IF $i \leq N$ THEN:

(a) COMPUTE MASS POSITION: $\boldsymbol{r}_{i}^{L+1, K}$

(b) GO TO (2) AND NEXT MASS $(i=i+1)$

(4) ERROR MEASURE:
(a) $\varpi_{K} \stackrel{\text { def }}{=} \frac{\sum_{i=1}^{N}\left\|\boldsymbol{r}_{i}^{L+1, K}-\boldsymbol{r}_{i}^{L+1, K-1}\right\|}{\sum_{i=1}^{N}\left\|\boldsymbol{r}_{i}^{L+1, K}-\boldsymbol{r}_{i}^{L}\right\|} \quad$ (normalized)
(b) $Z_{K} \stackrel{\text { def }}{=} \frac{\varpi_{K}}{T O L_{r}}$
(c) $\Phi_{K} \stackrel{\text { def }}{=}\left(\frac{\left(\frac{T O L}{\varpi_{0}}\right)^{\frac{1}{p K} d}}{\left(\frac{\varpi_{K}}{\varpi_{0}}\right)^{\frac{1}{p K}}}\right)$

(5) IF TOLERANCE MET $\left(Z_{K} \leq 1\right)$ AND $K<K_{d}$ THEN:

(a) CONSTRUCT NEW TIME STEP: $\Delta t=\Phi_{K} \Delta t$

(b) SELECT MINIMUM: $\Delta t=\operatorname{MIN}\left(\Delta t^{l i m}, \Delta t\right)$

(c) INCREMENT TIME: $t=t+\Delta t$ AND GO TO (1)

(6) IF TOLERANCE NOT MET $\left(Z_{K}>1\right) A N D K=K_{d}$ THEN:

(a) CONSTRUCT NEW TIME STEP: $\Delta t=\Phi_{K} \Delta t$

(b) RESTART AT TIME $=t$ AND GO TO (1)

Remark External damping can easily be modeled by adding $c_{i} \dot{\boldsymbol{r}}_{i}$ in the equations of motion:

$$
m_{i} \ddot{\boldsymbol{r}}_{i}=\underbrace{\boldsymbol{\psi}_{i}^{\text {tot }}}_{\text {total }}=\underbrace{\boldsymbol{\psi}_{i}^{\text {em }}}_{\text {electromagnetic forces }}+\underbrace{\sum_{I=1}^{4} \boldsymbol{\psi}_{i I}^{\text {yarn }}}_{\text {surrounding yarn }}-\underbrace{c_{i} \dot{\boldsymbol{r}}_{i}}_{\text {damping }} .
$$

\section{A Model Problem}

As a model problem, we consider the presence of a magnetic field, $\boldsymbol{B}^{\text {ext }}=B_{1}^{\text {ext }} \boldsymbol{e}_{1}$. Note that without an electric field to induce a velocity for the lumped-charges/masses, an initially stationary functionalized fabric will not deform, since

$$
m_{i} \dot{\boldsymbol{v}}_{i}=\boldsymbol{v}_{i} \times \boldsymbol{B}^{e x t}=\mathbf{0} .
$$

Furthermore, no deformation would occur for $\boldsymbol{B}^{\text {ext }}$-fields where the initial $\boldsymbol{v}_{i}$ and $\boldsymbol{B}^{\text {ext }}$ are parallel. As a model problem, the following system parameters were used:

- yarn radii $=0.0005$ (meters),

- lumped charged-masses: $50 \times 50$ (a $50 \times 50$ yarn-network weave),

- lumped mass charge $q=1$ (Coloumb),

- external magnetic field $\boldsymbol{E}^{\text {ext }}=1.0 \boldsymbol{e}_{3}$ (Newton/Coulomb), $\boldsymbol{B}^{\text {ext }}=0.1 \boldsymbol{e}_{1}$ (Newtonsecond/Coulomb-meter),

- trapezoidal time-stepping parameter $\phi=0.5$ (mid-point rule),

- area-based density of fabric $=0.15$ (kilograms $/$ meter $\left.^{2}\right)$, leading to lumped masses of $m_{i}=\frac{(0.254)^{2} \times 0.15}{(50)^{2}}($ kilograms $)$,

- damping coefficient, $c=0.01$ (Newton-second/meter), 
Fig. 4 A qualitative initial velocity profile inducing an increasingly larger Lorentz force along the symmetry line

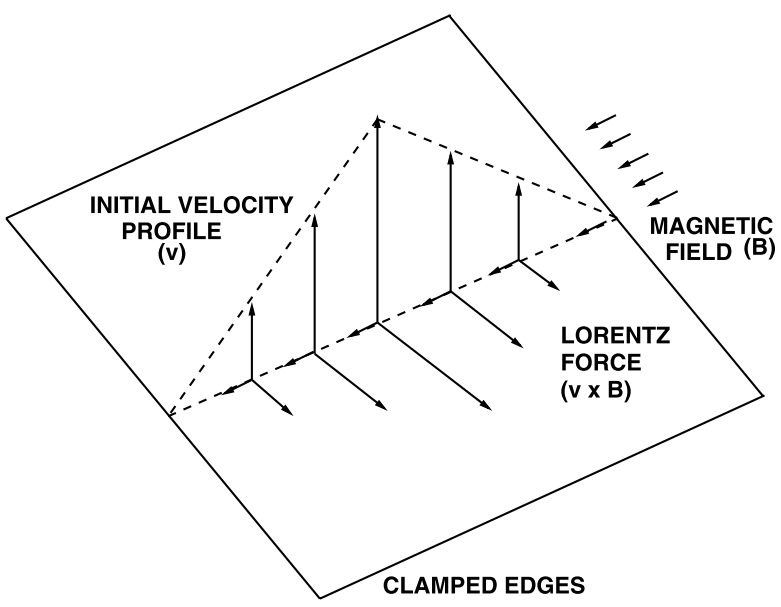

- size of the sheet: $0.254 \mathrm{~m} \times 0.254$ (meters) $(=10$ in $\times 10$ (inches)),

- average Young's modulus of a yarn-segment $\mathbb{E}=100$ (mega-Pascals) (a typical polymer), with a random statistical variation of $\pm 10 \%$ from the mean governed by a Gaussian distribution,

- initial (upper limit) time-step size: $\Delta t=0.000001$ (seconds),

- iterative tolerance per time step: $T O L=0.000001$,

- iteration limit per time-step: $K_{d}=6$.

The events connected to the case of having only an electric field present and the case of having both electric and magnetic fields present, can be observed in Figs. 6 and 7. In the first simulation, with no magnetic fields, the symmetric deformation is evident. ${ }^{5}$ In the presence of a combined electromagnetic field there is a break in "four-quadrant" symmetry due to the term $\boldsymbol{v}_{i} \times \boldsymbol{B}^{\text {ext }}$. Initially, since the fabric is clamped on all four sides, the electric field $\left(\boldsymbol{E}^{\text {ext }}\right.$ ) will produce a monotonically decreasing velocity profile from the system center point (Fig. 4). This will result in a larger $\boldsymbol{v}_{i} \times \boldsymbol{B}^{\text {ext }}$ contribution along the center (symmetry) line, resulting in a pulse in the middle of the $x_{2}$-direction (Figs. 4 and 7 )

$$
v_{o} \boldsymbol{e}_{3} \times B_{1}^{e x t} \boldsymbol{e}_{1}=v_{o} B_{1}^{e x t} \boldsymbol{e}_{2} .
$$

We remark that if the magnetic field had been applied in the other (in-plane) $x_{2}$-direction, then

$$
v_{o} \boldsymbol{e}_{3} \times B_{2}^{\text {ext }} \boldsymbol{e}_{2}=-v_{o} B_{2}^{\text {ext }} \boldsymbol{e}_{1} .
$$

The long-term dynamics of both simulations (electric field only and combined electric and magnetic fields) would arrive at the same steady-state response, since the presence of damping, via the term $c_{i} \dot{\boldsymbol{r}}_{i}$ in (41) eventually brings the system to a halt and, consequently, for each charged-mass, $\boldsymbol{v}_{i} \times \boldsymbol{B}^{\text {ext }}=\mathbf{0}$. It is also worth noting that the time for the transience to die down is significantly longer when the magnetic field is present, due to the $\boldsymbol{v}_{i} \times \boldsymbol{B}^{\text {ext }}$ term. The simulations take on the order of four to five minutes on a standard laptop and the

\footnotetext{
${ }^{5}$ Such a symmetry is not an exact symmetry since, as indicated earlier, the material has a random statistical variation of $\pm 10 \%$ from the mean governed by a Gaussian distribution.
} 
Fig. 5 Prestressing a segment of yarn
UNSTRETCHED
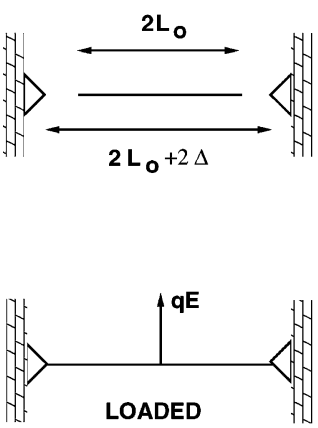

STRETCHED
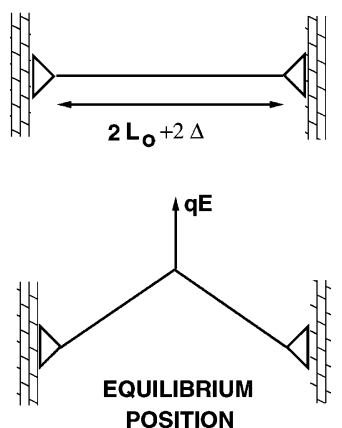

computational algorithm scales extremely well for much larger fabric systems (see Zohdi [46] and Zohdi and Powell [41]).

Remark 1 Although we have not included yarn damage in the present formulation, it is now appropriate to make some remarks on this issue. Generally, the microstructure of the fabric yarn is composed of microscale fibrils. This is the case with structural fabric materials such as Zylon, which is a polymeric material produced by the Toyobo Corporation (Toyobo [35]), Kevlar or other aramid-based materials. These materials have a multiscale structure, for example, constructed from PBO (Polybenzoxale) microscale fibrils, which are bundled together to form yarn, which are then tightly woven into sheets. For Zylon, each yarn contains approximately 350 microfibrils, which are randomly misaligned within the yarn, leading to a gradual type of failure, since the microfibrils become stretched to different lengths (within the yarn), when the yarn is in tension. A simple approach to describe failure of a yarn is to check whether a critical stretch has been attained or exceeded, $U(t) \geq U_{\text {crit }}$, and to track the progressive damage for a yarn with a single damage (isotropic) variable used in $S=\alpha \mathbb{E} \mathcal{E}$, where 0 (ruptured) $\leq \alpha \leq 1$ (undamaged). The damage variable for each yarn-segment typically has an evolution law associated with it, which represents progressive stretch-induced damage. ${ }^{6}$

Remark 2 The simulation of the actuation of electromagnetically sensitive networks for more sophisticated, constitutive relations requires only a replacement of the rudimentary constitutive relation in (3). For example, for networks of biological materials (see Zohdi [43]), one could use a one-dimensional Fung-like material model (or appropriate variant) for the yarn-segments (Fung [12]) with stored energy given by $W=c\left(e^{\eta}-1\right)$, where $\eta=$ $\frac{1}{2} H \mathcal{E}^{2}, H$ and $c$ are material constants, and where the second Piola-Kirchhoff stress is given by $S=\frac{\partial W}{\partial \mathcal{E}}=c \frac{\partial \eta}{\partial \mathcal{E}} e^{\eta}=c H \mathcal{E} e^{\frac{H \mathcal{E}^{2}}{2}}$.

\section{Summary and Extensions}

This work investigated the deformation of electromagnetically functionalized fabric via a reduced-order model comprised of an interconnected yarn-segment network combined with

\footnotetext{
${ }^{6}$ Multiscale and damage formulations for structural yarn have been explored in detail in Zohdi and Steigmann [37], Zohdi and Powell [41], Powell and Zohdi [29] and Zohdi [46].
} 

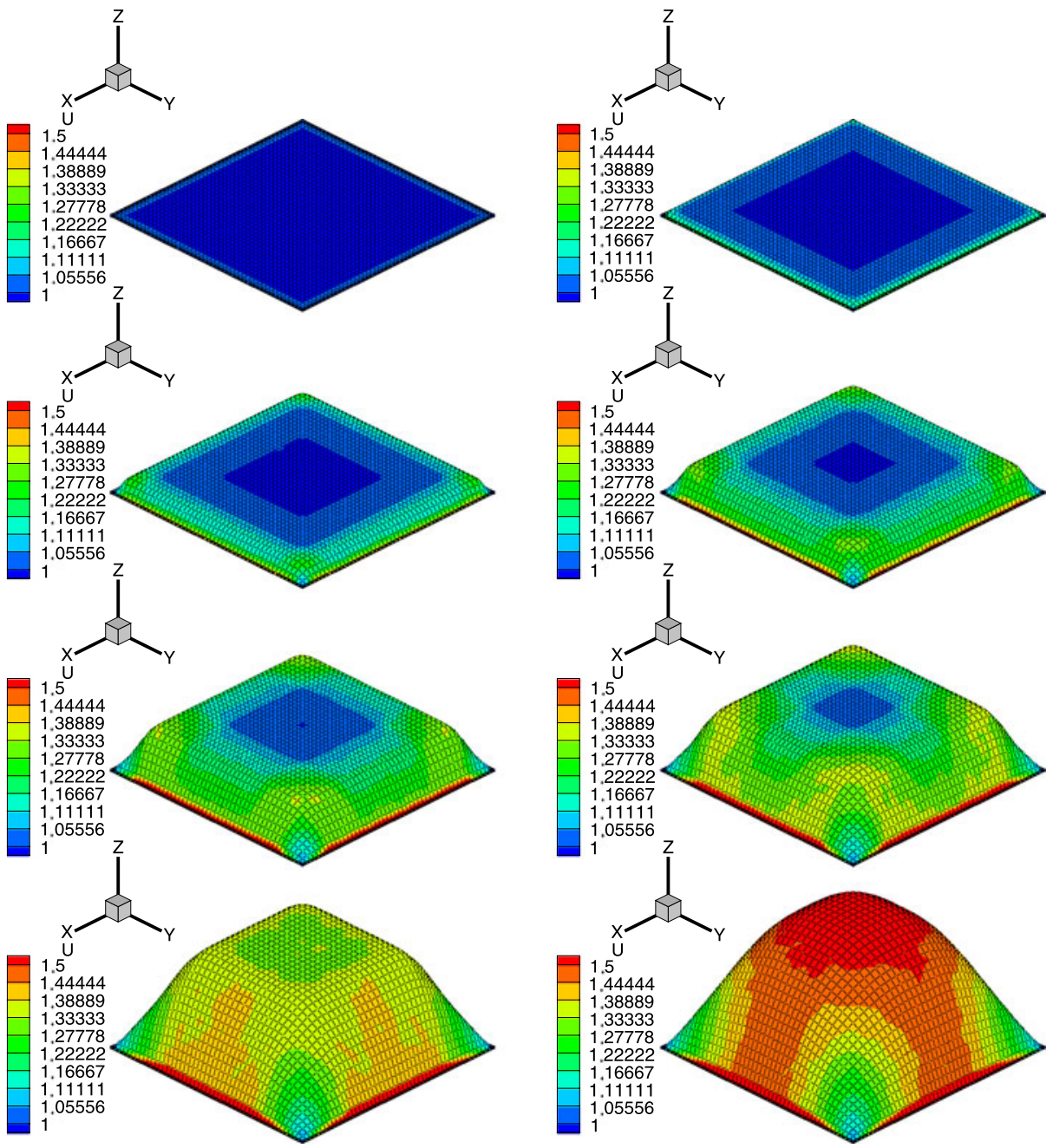

Fig. 6 Response, with only electric fields: Top to bottom and left to right: sequence of frames of the response of the fabric. The colors indicate the yarn-segment stretch $(U)$. The Young's modulus of the yarn have a random statistical variation of $\pm 10 \%$ from the mean governed by a Gaussian distribution

lumped charged-masses. The fabric deflection is dictated by solving a coupled system of differential equations for the motion of the fabric-coupled lumped charged-masses. The effects of the external electric and magnetic fields were analytically investigated and quantitative large-scale simulations were provided, based on a computationally-efficient time-stepping algorithm.

An important topic for further study is to incorporate the effects of prestressing/prestretching the fabric. For example, let us revisit a single yarn-segment unit, and consider the process depicted in Fig. 5. If we consider a prestretch of $2 \Delta$ (two yarn-segments attached), we see that the final stretched length of the yarn-segment is $2 L$, where

$$
L=\sqrt{\left(L_{o}+\Delta\right)^{2}+\delta^{2}} .
$$



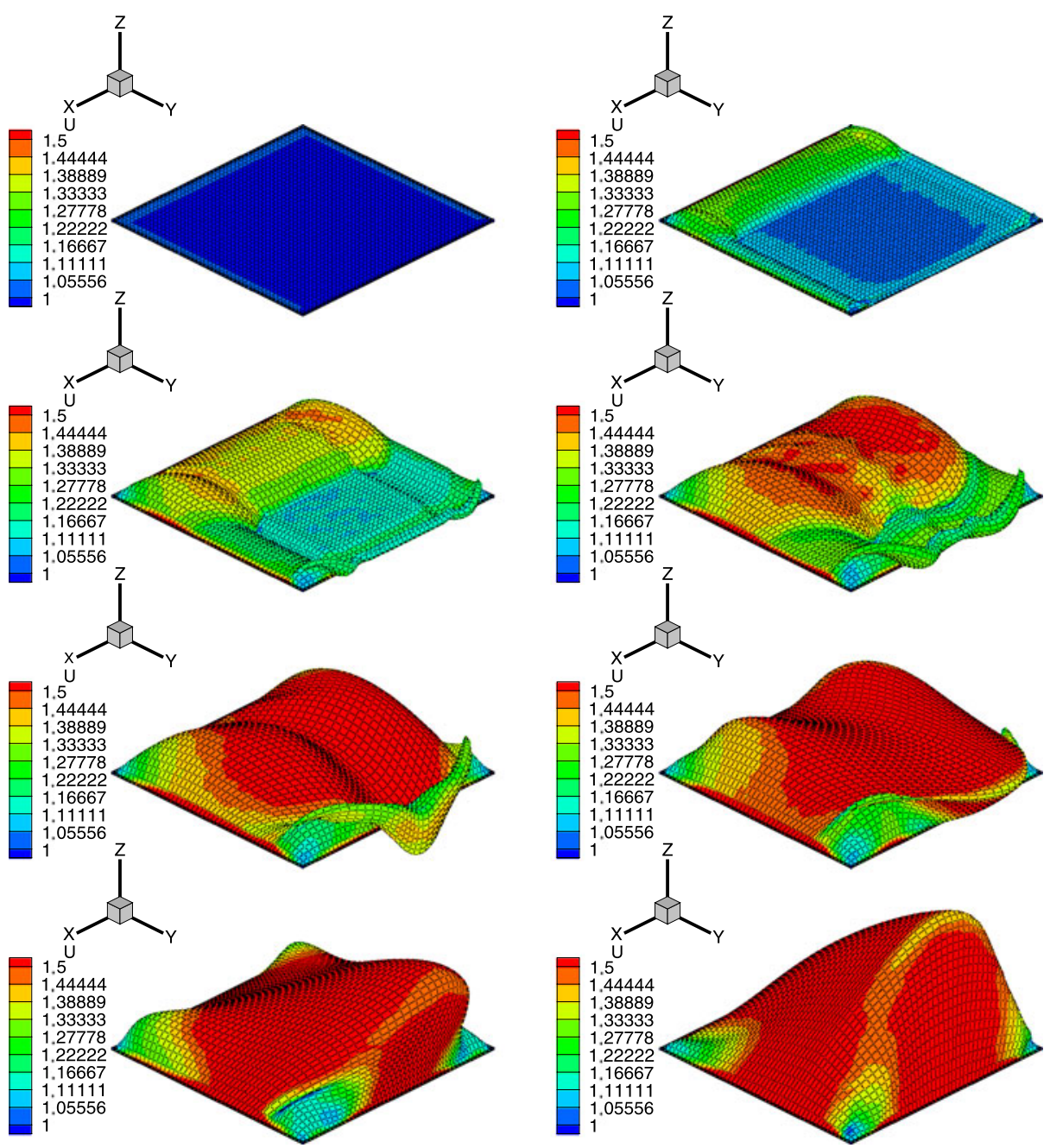

Fig. 7 Response, with both electric and magnetic fields: Top to bottom and left to right: sequence of frames of the response of the fabric. The colors indicate the yarn-segment stretch $(U)$. The Young's modulus of the yarn have a random statistical variation of $\pm 10 \%$ from the mean governed by a Gaussian distribution

Again performing a static equilibrium analysis, for a purely electrical field, we obtain the following relationship:

$$
q E^{e x t}=2 P A_{o} \frac{\delta}{L}=2 U S A_{o} \frac{\delta}{L}=\frac{L}{L_{o}} \mathbb{E}\left(\frac{L^{2}}{L_{o}^{2}}-1\right) A_{o} \delta=\frac{\mathbb{E} A_{o} \delta}{L_{o}^{3}}\left(2 \Delta L_{o}+\Delta^{2}+\delta^{2}\right) .
$$

The cubic equation is of the form,

$$
\delta^{3}+\delta \underbrace{\left(2 \Delta L_{o}+\Delta^{2}\right)}_{\substack{\text { def } \\=}}-\underbrace{\frac{q E^{e x t} L_{o}^{3}}{\mathbb{E} A_{o}}}_{\stackrel{q \text { def }}{=}-b}=0
$$


This equation has one real root, and two complex conjugate roots. If one solves the cubic equation for the real root, one obtains

$$
\delta=\left(-\frac{b}{2}+\sqrt{D}\right)^{\frac{1}{3}}+\left(-\frac{b}{2}-\sqrt{D}\right)^{\frac{1}{3}},
$$

where $D=\frac{b^{2}}{4}+\frac{a^{3}}{27}$. For $\Delta>0$, the prestretched fabric yarn are stiffer $(\delta(\Delta)<\delta(\Delta=0))$, but will reach their rupture stretch more quickly. Precompressing the yarn (producing "slack") can lead to a "trampoline" effect, which may be useful in certain applications. The effects of prestressing electromagnetic fabric is under current investigation by the author.

Finally, we close with some comments on the practical matter of charging the material. In this work we considered the charges to be static within the fabric, i.e., they were not flowing. For example, this type of "static" charge can delivered in the form of an ionimplantation/bombardment/spray onto the fabric. Furthermore, in some cases, one could consider materials that can be charged like a battery, provided that they have an inherent capacitance. An alternative way of delivering charge to the fabric could be achieved by running live current $(\boldsymbol{J})$ through the fabric system. This would lead to forces proportional to the flowing current crossed with the magnetic field $(\boldsymbol{J} \times \boldsymbol{B})$. Generally speaking, the current will depend on the deformation of the fabric, and may also produce an induced electromagnetic field (computable from the Biot-Savart relation). Without any simplifications, such a system must be treated by direct simulation of a fully coupled set of equations comprised of Maxwell's equations and the balance of momentum, which inevitably leads to non-trivial issues in numerical discretization and high-performance (large-scale) computing. Furthermore, depending on the type and level of actuation needed, the level of electromagnetism may induce thermal effects via Joule heating. A detailed account of computational methods to simulate these effects can be found in Zohdi [47] and is beyond the scope of the current paper. The development of reduced-order models that attempt to capture the essential features of such a electromagnetic delivery system, without resorting to full-scale computations, are under current investigation by the author.

Acknowledgements This work was funded in part by the Army Research Laboratory through the Army High Performance Computing Center (cooperative agreement W911NF-07-20027) and the Powley foundation.

Open Access This article is distributed under the terms of the Creative Commons Attribution Noncommercial License which permits any noncommercial use, distribution, and reproduction in any medium, provided the original author(s) and source are credited.

\section{References}

1. Atai, A.A., Steigmann, D.J.: On the nonlinear mechanics of discrete networks. Arch. Appl. Mech. 67, 303-319 (1997)

2. Atai, A.A., Steigmann, D.J.: Coupled deformations of elastic curves and surfaces. Int. J. Solids Struct. 35(16), 1915-1952 (1998)

3. Azevedo, R.G., Jones, D.G., Jog, A.V., Jamshidi, B., Myers, D.R., Chen, L., Fu, X.A., Mehregany, M., Wijesundara, M.B.J., Pisano, A.P.: A SiC MEMS resonant strain sensor for Harsh Environment applications. IEEE Sens. J. 7(4), 568-576 (2007)

4. Barham, M., Steigmann, D.J., McElfresh, M., Rudd, R.E.: Finite deformation of a pressurized magnetoelastic membrane in a stationary dipole field. Acta Mech. 191, 1-19 (2007)

5. Barham, M., Steigmann, D.J., McElfresh, M., Rudd, R.E.: Limit-point instability of a magnetoelastic membrane in a stationary magnetic field. J. Smart Mater. Struct. 17, 6-11 (2008) 
6. Barham, M., White, D., Steigmann, D.J., Rudd, R.E.: Finite-element modeling of the deformation of a thin magnetoelastic film compared to a membrane model. IEEE Trans. Magn. 45, 4124-27 (2009)

7. Barham, M., White, D., Steigmann, D.J.: Finite-element modeling of the deformation of magnetoelastic film. J. Comput. Phys. 229, 6193-6207 (2010)

8. Buchholdt, H.A., Davies, M., Hussey, M.J.L.: The analysis of cable nets. J. Inst. Math. Appl. 4, 339-358 (1968)

9. Bufler, H., Nguyen-Tuong, B.: On the work theorems in nonlinear network theory. Ing. Archit. 49, 275286 (1980)

10. Cannarozzi, M.: Stationary and extremum variational formulations for the elastostatics of cable networks. Meccanica 20, 136-143 (1985)

11. Cannarozzi, M.: A minimum principle for tractions in the elastostatics of cable networks. Int. J. Solids Struct. 23, 551-568 (1987)

12. Fung, Y.C.: On the foundations of biomechanics. ASME J. Appl. Mech. 50, 1003-1009 (1983)

13. Grimes, C., Ong, K., Loiselle, K., Stoyanov, P., Kouzoudis, D., Liu, Y., Tong, C., Tefiku, F.: Magnetoelastic sensors for remote query environmental monitoring. Smart Mater. Struct. 8, 639-646 (1999)

14. Haseganu, E.M., Steigmann, D.J.: Analysis of partly wrinkled membranes by the method of dynamic relaxation. Comput. Mech. 14, 596-614 (1994)

15. Haseganu, E.M., Steigmann, D.J.: Theoretical flexural response of a pressurized cylindrical membrane. Int. J. Solids Struct. 31, 27-50 (1994)

16. Haseganu, E.M., Steigmann, D.J.: Equilibrium analysis of finitely deformed elastic networks. Comput. Mech. 17, 359-373 (1996)

17. Hashin, Z.: Analysis of composite materials: a survey. ASME J. Appl. Mech. 50, 481-505 (1983)

18. Jackson, J.D.: Classical Electrodynamics, 3rd edn. Wiley, New York (1998)

19. Jikov, V.V., Kozlov, S.M., Olenik, O.A.: Homogenization of Differential Operators and Integral Functionals. Springer, Berlin (1994)

20. Jones, D.G., Azevedo, R.G., Chan, M.W., Pisano, A.P., Wijesundara, M.B.J.: Low temperature ion beam sputter deposition of amorphous silicon carbide for vacuum encapsulation. In: Proceedings of the IEEE International Conference on Micro Electro Mechanical Systems, Kobe, Japan (2007)

21. Kouzoudis, D., Grimes, C.: The frequency response of magnetoelastic sensors to stress and atmospheric pressure. Smart Mater. Struct. 9, 885-889 (2000)

22. Kouzoudis, D., Grimes, C.: Remote query fluid flow velocity measurement using magnetoelastic thick film sensors. J. Appl. Phys. 87(9), 6301-6303 (2000)

23. Maxwell, J.C.: On the dynamical theory of gases. Philos. Trans. Soc. Lond. 157, 49 (1867)

24. Maxwell, J.C.: A Treatise on Electricity and Magnetism, 3rd edn. Clarendon Press, Oxford (1873)

25. Myers, D.R., Cheng, K.B., Jamshidi, B., Azevedo, R.G., Senesky, D.G., Chen, L., Mehregany, M., Wijesundara, M.B.J., Pisano, A.P.: A silicon carbide resonant tuning fork for micro-sensing applications in high temperature and high G-shock environments. J. Micromech. Microeng. (2009)

26. Nemat-Nasser, S., Hori, M.: Micromechanics: Overall Properties of Heterogeneous Solids, 2nd edn. Elsevier, Amsterdam (1999)

27. Pangiotopoulos, P.D.: A variational inequality approach to the inelastic stress-unilateral analysis of cable structures. Comput. Struct. 6, 133-139 (1976)

28. Pipkin, A.C.: The relaxed energy density for isotropic elastic membranes. IMA J. Appl. Math. 36, 297308 (1986)

29. Powell, D., Zohdi, T.: Attachment mode performance of network-modeled ballistic fabric shielding. Composites Part B 40, 451-460 (2009)

30. Rayleigh, J.W.: On the influence of obstacles arranged in rectangular order upon properties of a medium. Philos. Mag. 32, 481-491 (1892)

31. Quandt, E., Ludwig, A.: Magnetorestrictive actuation in microsystems. Sens. Actuators 81, 275-280 (2000)

32. Rebeiz, G.M., Barker, N.S., Muldavin, J.B., Tan, G.-L.: Mechanical Modeling of MEMS Devices: Static Analysis RF MEMS: Theory, Design and Technology, pp. 21-57. Wiley, New York (2004). Ed. G.M. Rebeiz

33. Steigmann, D.J.: Tension field theory. Proc. R. Soc. Lond. A 429, 141-173 (1990)

34. Torquato, S.: Random Heterogeneous Materials: Microstructure and Macroscopic Properties. Springer, New York (2001)

35. Toyobo: PBO fiber ZYLON. Report of the Toyobo Corporation, LTD (2001). www.toyobo.co.jp

36. Zohdi, T.I.: Modeling and simulation of progressive penetration of multilayered ballistic fabric shielding. Comput. Mech. 29, 61-67 (2002)

37. Zohdi, T.I., Steigmann, D.J.: The toughening effect of microscopic filament misalignment on macroscopic fabric response. Int. J. Fract. 115, L9-L14 (2002) 
38. Zohdi, T.I.: Modeling and direct simulation of near-field granular flows. Int. J. Solids Struct. 42(2), 539564 (2004)

39. Zohdi, T.I.: A computational framework for agglomeration in thermo-chemically reacting granular flows. Proc. R. Soc. 460(2052), 3421-3445 (2004)

40. Zohdi, T.I.: Charge-induced clustering in multifield particulate flow. Int. J. Numer. Methods Eng. 62(7), 870-898 (2005)

41. Zohdi, T.I., Powell, D.: Multiscale construction and large-scale simulation of structural fabric undergoing ballistic impact. Comput. Methods Appl. Mech. Eng. 195(1-3), 94-109 (2006)

42. Zohdi, T.I.: Computation of strongly coupled multifield interaction in particle-fluid systems. Comput. Methods Appl. Mech. Eng. 196, 3927-3950 (2007)

43. Zohdi, T.I.: A computational framework for network modeling of fibrous biological tissue deformation and rupture. Comput. Methods Appl. Mech. Eng. 196, 2972-2980 (2007)

44. Zohdi, T.I.: Introduction to the Modeling and Simulation of Particulate Flows. SIAM, Philadelphia (2007)

45. Zohdi, T.I.: Dynamics of clusters of charged particulates in electromagnetic fields. Int. J. Numer. Methods Eng. (2010, in press)

46. Zohdi, T.I.: High-speed impact with electromagnetically sensitive fabric and induced projectile spin. Comput. Mech. 46, 399-415 (2010)

47. Zohdi, T.I.: Simulation of coupled microscale multiphysical-fields in particulate-doped dielectrics with staggered adaptive FDTD. Comput. Methods Appl. Mech. Eng. 199, 79-101 (2010)

48. Zohdi, T.I., Wriggers, P.: Introduction to Computational Micromechanics, 2nd reprinting. Springer, Berlin (2008) 